\title{
Genetic, Clinicopathological, and Radiological Features of Intrahepatic Cholangiocarcinoma with Ductal Plate Malformation Pattern
}

\author{
Taek Chung ${ }^{1}$, Hyungjin Rhee ${ }^{2}$, Hyo Sup Shim³ ${ }^{3}$ Jeong Eun Yoo ${ }^{3}$, Gi Hong Choi ${ }^{4}$, Haeryoung Kim ${ }^{5}$, and Young \\ Nyun Park ${ }^{3,6}$ \\ ${ }^{1}$ Department of Biomedical Systems Informatics, ${ }^{2}$ Department of Radiology, Research Institute of Radiological Science, Center \\ for Clinical Imaging Data Science, Departments of ${ }^{3}$ Pathology and ${ }^{4}$ Surgery, Yonsei University College of Medicine, ${ }^{5}$ Department \\ of Pathology, Seoul National University Hospital, Seoul National University College of Medicine, and ${ }^{6}$ Graduate School of Medical \\ Science, Brain Korea 21 Project, Yonsei University College of Medicine, Seoul, Korea
}

\section{Article Info}

Received April 16, 2021

Revised July 9, 2021

Accepted July 15, 2021

Published online November 23, 2021

Corresponding Author

Young Nyun Park

ORCID https://orcid.org/0000-0003-0357-7967

E-mail young0608@yuhs.ac
Background/Aims: Intrahepatic cholangiocarcinoma (iCCA) with a ductal plate malformation (DPM) pattern is a recently recognized rare variant. The genomic profile of iCCA with DPM pattern needs to be elucidated.

Methods: Cases of iCCA with DPM pattern were retrospectively reviewed based on the medical records, pathology slides, and magnetic resonance imaging (MRI) reports collected between 2010 to 2019 at a single center. Massive parallel sequencing was performed for $>500$ cancerrelated genes.

Results: From a total of 175 iCCAs, five $(2.9 \%)$ cases of iCCA with DPM pattern were identified. All cases were of the small duct type, and background liver revealed chronic B viral or alcoholic hepatitis. Three iCCAs with DPM pattern harbored MRI features favoring the diagnosis of hepatocellular carcinoma, whereas nonspecific imaging features were observed in two cases. All patients were alive without recurrence during an average follow-up period of 57 months. Sequencing data revealed 64 mutated genes in the five cases, among which FGFR2 and PTPRT were most frequently mutated (three cases each) including an FGFR2-TNC fusion in one case. Mutations in ARID1A and CDKN2A were found in two cases, and mutations in TP53, BAP1, ATM, NF1, and STK11 were observed in one case each. No IDH1, KRAS, or PBRM1 mutations were found.

Conclusions: iCCAs with DPM pattern have different clinico-radio-pathologic and genetic characteristics compared to conventional iCCAs. Moreover, FGFR2 and ARID1A variants were identified. Altogether, these findings further suggest that iCCA with DPM pattern represents a specific subtype of small duct type iCCA. (Gut Liver 2022;16:613-624)

Key Words: Cholangiocarcinoma; High-throughput nucleotide sequencing; Immunohistochemistry; Magnetic resonance imaging

\section{INTRODUCTION}

Intrahepatic cholangiocarcinoma (iCCA) is a malignant neoplasm characterized by biliary differentiation. It is the second most common liver cancer worldwide, constituting approximately $15 \%$ of all primary liver malignancies. ${ }^{1}$ The histomorphology of iCCA is diverse, and a rare variant of iCCA with ductal plate malformation (DPM) pattern has recently been reported. ${ }^{2}$ DPM is a developmental anomaly, resulting in the partial persistence of redundant embryonic bile duct structures, known as ductal plates, which often represent elongated, tortuous, and coalesced bile duct-like structures in fibrous stroma. ${ }^{3,4}$ iCCA with DPM pattern shows histopathological features similar to DPM, in which tumor epithelial components are composed of glandular structures with irregularly dilated lumens, and tumor cells showing a low-columnar-to-cuboidal shape with little or no mucin production. ${ }^{2}$ iCCA with DPM pattern was re- 
cently recognized by the World Health Organization as a novel histopathological subtype of iCCA; however, its genetic characteristics remain unclear. ${ }^{5}$

Several recent studies focused on the altered genomic features of iCCA identified multiple putative driver mutations using massive parallel sequencing methods (also called next-generation sequencing or NGS) ${ }^{6,7}$ Frequently mutated genes associated with iCCA include known tumor suppressor genes, such as TP53, ARID1A, FGFR2, and $B A P 1$, as well as oncogenes, such as IDH1 and KRAS. ${ }^{7-10}$ Although the genetic features of iCCA related to various histopathological features remain unclear, some mutations were reported to be associated with specific histopathological features. KRAS and TP53 mutations were reported to be associated with large (bile) duct type, ${ }^{11}$ and $I D H 1 / 2 \mathrm{mu}$ tations and FGFR fusions were more frequently found in small duct type than in other histological iCCA types. ${ }^{10,12,13}$

Herein, the genetic profile of iCCA with DPM pattern evaluated by NGS is presented along with a clinicopathological and radiological review of five patients.

\section{MATERIALS AND METHODS}

\section{Case selection and clinicopathological data}

The electronic medical records of patients who underwent curative liver resection at Severance Hospital, Seoul, Republic of Korea, and who were diagnosed with iCCA between January 2010 and December 2019 were reviewed. Among the 175 iCCA cases identified during this time period, a total of five (2.9\%) cases of iCCA with DPM pattern were found and confirmed upon the assessment of their hematoxylin and eosin stained tumor section slides. The presence and predominance ( $>90 \%$ of the tumor area) of the DPM pattern were confirmed in all five cases. Demographic and clinical data of the patients, including age, sex, preoperative serum chemistry, tumor markers, and postoperative follow-up records, were collected. All patients were routinely followed up for surveillance of tumor recurrence using computed tomography. This study was approved by the Institutional Review Board of Severance Hospital (IRB number: 4-2020-0286), and the requirement for informed consent was waived.

\section{Dynamic contrast-enhanced MRI assessment}

In three cases, magnetic resonance imaging (MRI) was performed using two 3.0-T systems at our institution (cases no. 1 and no. 2 in MAGNETOM Trio Tim, Siemens Healthineers, Erlangen, Germany; case no. 3 in Intera Achieva, Philips Medical Systems, Amsterdam, The Netherlands). In the remaining two cases (cases no. 4 and no. 5),
MRIs were performed in the hospital where the patients were referred using a 3.0-T system (MAGNETOM Skyra; Siemens Healthineers). In all cases, gadoxetic acid disodium (Bayer Schering Pharma, Berlin, Germany) was used as contrast agent for dynamic imaging. Three-dimensional gradient echo sequences with chemical-selective fat suppression were acquired before and after an intravenous injection of gadoxetic acid, using a section thickness, repetition time, and echo time of 2-3 mm, 2.54-4.14 milliseconds, and 0.95-1.71 milliseconds, respectively. After obtaining the precontrast images, contrast-enhanced dynamic and hepatobiliary phase images were acquired from the arterial, portal, transitional, and hepatobiliary phases at approximately $25-30,65-75$, and $130-150$ seconds, and 15-20 minutes after contrast injection, respectively. Between the dynamic and hepatobiliary phases, $\mathrm{T} 2$-weighted (spin-echo sequences using a navigator-triggered technique) and diffusion-weighted images (navigator-triggered technique) were obtained.

The MRI images were analyzed by an experienced faculty abdominal radiologist (H.R.) and were evaluated according to the Liver Imaging Reporting and Data System (LI-RADS) (version 2018), ${ }^{14}$ including assessment of major features (non-rim arterial phase hyperenhancement, enhancing capsule, and non-peripheral washout), ancillary features (restricted diffusion, T2 hyperintensity, fat sparing, iron sparing, transitional phase hypointensity, hepatobiliary phase hypointensity, non-enhancing capsule, nodulein-nodule, mosaic architecture, blood product in mass, and fat in mass), and LR-M features (rim arterial phase hyperenhancement, peripheral washout, delayed central enhancement in hepatobiliary phase, targetoid restriction, and targetoid transitional phase or hepatobiliary phase appearance). Additionally, capsular retraction and peritumoral shunt were evaluated. The peritumoral shunt was defined as a detectable portion of a crescent or polygonal enhancement outside the tumor margin with broad contact with the tumor border in the arterial or portal phase, becoming isointense with background liver parenchyma in the delayed phase. LI-RADS categorization was applied in high-risk patients for hepatocellular carcinoma (HCC) as follows: (1) cirrhosis, (2) chronic hepatitis B viral infection, or (3) current or prior HCC.

\section{Immunohistochemical and special staining}

Representative whole-section slides of the tumors were immunohistochemically stained for $\mathrm{N}$-cadherin (1:100, mouse monoclonal; Zymed Laboratories Inc., San Francisco, CA, USA), neural cell adhesion molecule (1:100, mouse monoclonal; Leica Biosystems, Nussloch, Germany), S100 calcium-binding protein $\mathrm{P}$ (1:100, mouse monoclonal; 
R\&D Systems, Minneapolis, MN, USA), CD3 (1:100, mouse polyclonal; Dako, Glostrup, Denmark), CD4 (prediluted, mouse monoclonal; Dako), CD8 (prediluted, mouse monoclonal; Leica Biosystems), CD68 (1:300, mouse monoclonal; Dako), CD163 (1:50, mouse monoclonal; Cell Marque, Rocklin, CA, USA), FOXP3 (1:100, mouse monoclonal; Abcam, Cambridge, MA, USA), C-reactive protein (rabbit monoclonal; Abcam), and programmed death-ligand 1 (mouse monoclonal; Dako) using an automated staining system (Ventana Medical Systems, Tucson, AZ, USA) according to the manufacturer's instructions. The amount of immune cell infiltration was counted in 10 high-power field-equivalent area from microscopic images by QuPath software (University of Edinburgh, Edinburgh, UK). ${ }^{15}$ Alcian blue staining was also performed to evaluate the presence and extent of intra/extracellular mucin production.

\section{NGS and data analysis}

Formalin-fixed, paraffin-embedded tissues were obtained from the Liver Cancer Specimen Bank (part of the National Research Bank Program, Korea Science and Engineering Foundation, Ministry of Science and Technology). Genomic DNA and RNA were extracted using the AllPrep DNA/RNA FFPE Kit (Qiagen, Hilden, Germany), according to the manufacturer's instructions. The TruSight Oncology 500 gene panel (Illumina, San Diego, CA, USA) was used for library preparation and hybrid capture of 523 genes and 55 transcripts from the DNA and RNA samples, respectively. Sequencing of each sample was performed using the NextSeq 550Dx System (Illumina), generating FASTQ files. Variant calling was performed using the TruSight Oncology 500 Local App (Illumina, version 1.3.1.3), which contains pipelines for the analysis of FASTQ files from DNA and RNA samples. Briefly, BWA-mem and SAMtools were used for DNA sequence alignment with the reference genome (GRCh37/hg19). ${ }^{16}$ Aligned reads in sequence alignment/map format were further processed, and variant calling was performed using the Pisces software. ${ }^{17}$ Annotation and initial filtering of called variants in variant call format file was performed with the Illumina Annotation Engine using the Nirvana software (https://github. com/Illumina/Nirvana/wiki). Variants with frequencies $<1 \%$ of the population, according to the Genome Aggregation Database (https://gnomad.broadinstitute.org) and Catalogue of Somatic Mutations In Cancer (https://cancer. sanger.ac.uk/cosmic), were selected for further analysis. Variants listed in the Korean Reference Genome Database (https://nih.go.kr/contents.es? $\mathrm{mid}=\mathrm{a} 50303020400$ ) were also filtered out. RNA analysis included fusion calling, splice variant calling, and annotation of the results.

\section{RESULTS}

\section{Clinico-radiological features of iCCAs with DPM pattern}

All five patients diagnosed with iCCAs with DPM pattern were women, with an average age of 67 years at diagnosis, among whom four (80\%) were positive for hepatitis $B$ virus infection. Serum alpha-fetoprotein levels were not elevated in all patients. Protein induced by vitamin $\mathrm{K}$ absence or antagonists-II levels were slightly elevated in one case (case no. 4, $43 \mathrm{mAU} / \mathrm{mL}$ ), which had a separate mass of HCC. Additional markers were tested in only one case, showing no increase in cancer antigen 19-9 and carcinoembryonic antigen (Table 1).

Gadoxetic acid-enhanced MRI findings of iCCAs with DPM pattern are summarized in Table 2. Four cases (cases no. 1, 2, 3, and 4) showed non-rim arterial phase hyperenhancement. Cases no. 1 and no. 2 showed non-peripheral washout, and case no. 2 also exhibited an enhancing capsule. Collectively, the majority $(4 / 5,80 \%)$ of iCCA cases with DPM pattern demonstrated at least one major feature of LI-RADS, which favored HCC. In contrast, LR-M features which favored non-HCC malignancy were less fre-

Table 1. Demographic and Clinical Characteristics of the Five Cases Diagnosed with Intrahepatic Cholangiocarcinoma with Ductal Plate Malformation Pattern

\begin{tabular}{|c|c|c|c|c|c|c|c|c|c|}
\hline Case No. & $\begin{array}{l}\text { Age, yr/ } \\
\text { sex }\end{array}$ & Etiology & $\begin{array}{l}\text { Preoperative clinical } \\
\text { impression }\end{array}$ & $\begin{array}{l}\text { CA19-9, } \\
\mathrm{U} / \mathrm{mL}\end{array}$ & $\begin{array}{l}\text { CEA, } \\
\mathrm{ng} / \mathrm{mL}\end{array}$ & $\begin{array}{l}\text { AFP, } \\
I U / m L\end{array}$ & $\begin{array}{l}\text { PIVKA-II, } \\
\mathrm{mAU} / \mathrm{mL}\end{array}$ & $\begin{array}{l}\text { Follow-up } \\
\text { period, mo }\end{array}$ & Status \\
\hline 1 & $71 / F$ & HBV & $\mathrm{HCC}$ & 29.8 & 0.99 & 2.71 & 20 & 116 & NED \\
\hline 2 & $58 / F$ & Alcohol & $\mathrm{HCC}$ & NA & NA & 2.18 & 23 & 122 & NED \\
\hline 3 & $77 / F$ & HBV & $\mathrm{HCC}$ & NA & NA & 0.30 & 17 & 49 & NED \\
\hline $4^{*}$ & $67 / F$ & HBV & $\mathrm{HCC}$ & NA & NA & 2.73 & 43 & 27 & NED \\
\hline 5 & $60 / F$ & HBV & CHCC-CCA & NA & NA & 1.99 & 35 & 22 & NED \\
\hline
\end{tabular}

CA19-9, cancer antigen 19-9; CEA, carcinoembryonic antigen; AFP, alpha-fetoprotein; PIVKA-II, protein induced by vitamin $\mathrm{K}$ absence or antagonists-II; F, female; HBV, hepatitis B virus; HCC, hepatocellular carcinoma; cHCC-CCA, combined hepatocellular-cholangiocarcinoma; NA, not available; NED, no evidence of disease.

${ }^{*}$ A separate mass was concurrently found and resected, which was pathologically an HCC. 
Table 2. Gadoxetic Acid-Enhanced Magnetic Resonance Imaging Findings in Intrahepatic Cholangiocarcinomas with Ductal Plate Malformation Pattern

\begin{tabular}{|c|c|c|c|c|c|}
\hline \multirow{2}{*}{ Magnetic resonance imaging feature } & \multicolumn{5}{|c|}{ Case No. } \\
\hline & 1 & 2 & 3 & 4 & 5 \\
\hline High risk for $\mathrm{HCC}$ & + & - & + & + & + \\
\hline \multicolumn{6}{|l|}{ Major features (favoring $\mathrm{HCC}$ ) } \\
\hline Non-rim AP hyperenhancement & + & + & + & + & - \\
\hline Enhancing capsule & - & + & - & - & - \\
\hline Non-peripheral washout (PP) & + & + & - & - & - \\
\hline \multicolumn{6}{|l|}{ LR-M features (favoring non-HCC malignancy) } \\
\hline Rim AP hyperenhancement & - & - & - & - & + \\
\hline Peripheral washout & - & - & - & - & - \\
\hline Delayed central enhancement in HBP & - & - & - & - & - \\
\hline Targetoid restriction & - & - & - & - & - \\
\hline Targetoid TP or HBP appearance & - & - & - & - & - \\
\hline \multicolumn{6}{|l|}{ Ancillary features (favoring malignancy in general) } \\
\hline Restricted diffusion & + & + & + & + & + \\
\hline T2 hyperintensity & + & + & + & + & + \\
\hline Fat sparing & + & + & - & - & - \\
\hline Iron sparing & - & - & - & - & - \\
\hline TP hypointensity & + & + & - & + & + \\
\hline HBP hypointensity & + & + & + & + & + \\
\hline \multicolumn{6}{|l|}{ Ancillary features (favoring $\mathrm{HCC}$ ) } \\
\hline Non-enhancing capsule & - & - & - & - & - \\
\hline Nodule-in-nodule & - & - & - & - & - \\
\hline Mosaic architecture & - & - & - & - & - \\
\hline Blood product in mass & - & - & - & - & - \\
\hline Fat in mass & - & - & - & - & - \\
\hline LI-RADS category & LR-5 & NA & LR-4 & LR-M & LR-M \\
\hline \multicolumn{6}{|l|}{ Other features } \\
\hline Capsular retraction & - & - & - & + & - \\
\hline Peritumoral shunt & - & $\mathrm{AP}$ & $A P, P P$ & $\mathrm{PP}$ & $\mathrm{AP}$ \\
\hline
\end{tabular}

HCC, hepatocellular carcinoma; AP, arterial phase; PP, portal phase; HBP, hepatobiliary phase; TP, transitional phase; LI-RADS, Liver Imaging Reporting and Data System; NA, not available.

quently observed in the iCCA with DPM cases. Only case no. 5 demonstrated a rim arterial phase hyperenhancement. Other LR-M features, including peripheral washout, delayed central enhancement in the hepatobiliary phase, targetoid restriction, and targetoid transitional phase or hepatobiliary phase appearance, were not observed in any of the cases. Case no. 4 showed capsular retraction, which could be a clue for the diagnosis of iCCA. The iCCAs with DPM pattern also showed ancillary features favoring malignancy in general, including restricted diffusion $(5 / 5,100 \%), \mathrm{T} 2$ hyperintensity $(5 / 5,100 \%)$, fat-sparing $(2 / 5,40 \%)$, transitional phase hypointensity $(4 / 5,80 \%)$, and hepatobiliary phase hypointensity $(5 / 5,100 \%)$. Other ancillary features, including iron sparing, non-enhancing capsule, nodule-in-nodule, mosaic architecture, blood product in mass, and fat in in mass were not observed.

LI-RADS categorization could be applied to four patients (no. 1 , no 3 , no. 4 , and no. 5) who were at high risk for HCC. Specifically, case no. 1 was LR-5, case no. 3 was LR-4, and cases no. 4 and no. 5 were LR-M. Case no. 4 did not show any LR-M features; however, it was classified as LR-M due to the presence of capsular retraction. In the original preoperative radiology report, the primary diagnosis was HCC in three cases (cases no. 1, no. 2, and no. 3) and malignancy not specific for HCC (LR-M) in two cases (cases no. 4 and no. 5). Fig. 1 describes the radiological findings of two representative cases (cases no. 3 and no. 5) that were radiologically similar to and not specific for HCC, respectively.

A peritumoral shunt was frequently observed in iCCAs with DPM pattern. Arterial phase peritumoral shunt was noted in three cases (cases no. 2, no. 3, and no. 5), and portal phase peritumoral shunt was noted in two cases (cases no. 3 and no. 4).

Following clinical and radiological evaluation, preoperative clinical impression for four patients was HCC (cases no. 1 , no. 2, no. 3 , and no. 4). The clinical impression for case no. 5 was combined HCC-cholangiocarcinoma or iCCA. 

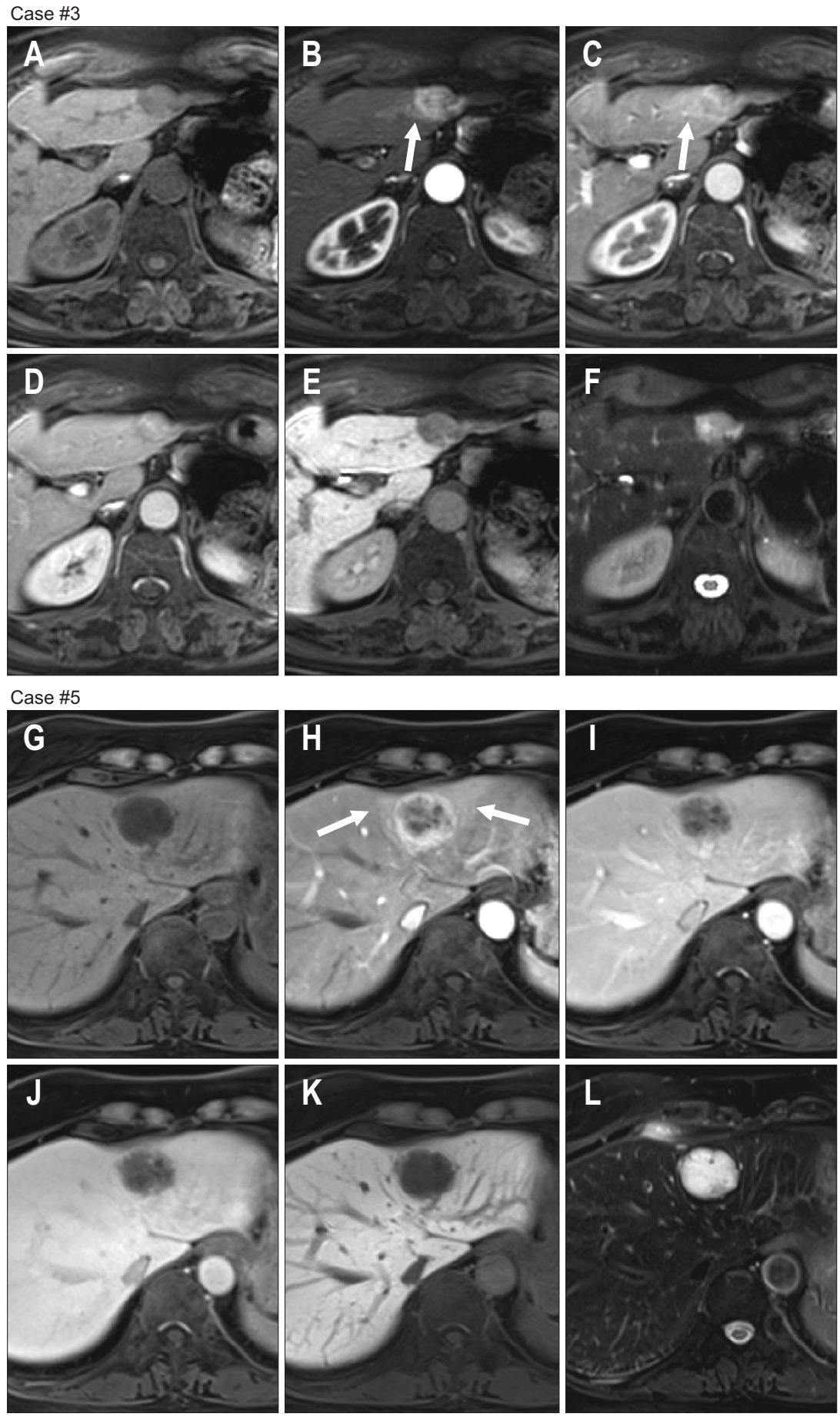

Fig. 1. Representative gadoxetic acid-enhanced magnetic resonance imaging (MRI) findings of two cases of intrahepatic cholangiocarcinoma with ductal plate malformation pattern. (A-F) Case no. 3, a 77-year-old female with hepatitis B virus-related chronic hepatitis showing imaging findings similar to hepatocellular carcinoma. A small hepatic lesion in liver segment 3 exhibited low-signal intensity in precontrast T1-weighted imaging (A), non-rim hyperenhancement in the arterial phase (B), and iso-signal intensity in the portal phase (C) and transitional phase (D) of gadoxetic acid-enhanced MRI. Peritumoral shunt was observed in the arterial phase and portal phase images (arrow). The lesion also showed hypo-signal intensity in the hepatobiliary phase (E) and hypersignal intensity in the T2 weighted image (F). Based on the imaging findings, the lesion could be categorized as LR-4 (probable hepatocellular carcinoma). (G-L) Case no. 5, a 60-year-old female with hepatitis B virus-related chronic hepatitis showing rim-like enhancement. A mass lesion of approximately $3 \mathrm{~cm}$ in liver segment 3 exhibited low-signal intensity in the precontrast T1weighted imaging $(\mathrm{G})$, rim-like hyperenhancement in the arterial phase $(\mathrm{H})$, and hypo-signal intensity in the portal phase $(\mathrm{I})$ and transitional phase (J) of gadoxetic acid-enhanced MRI. Peritumoral shunt was observed in the arterial phase image (arrows). The lesion also showed hypo-signal intensity in the hepatobiliary phase (K) and hypersignal intensity in the T2 weighted image (L). Due to rim-like arterial phase enhancement, the lesion was categorized as LR-M. 


\section{Pathological findings of iCCAs with DPM pattern and follow-up data}

Pathological examination of the resected specimen showed that all tumors were grossly mass-forming type with sizes ranging $1.5 \mathrm{~cm}$ to $3.6 \mathrm{~cm}, 2.7 \mathrm{~cm}$ on average. The histopathological features of the tumor resembled DPM in more than $90 \%$ of the tumor area. Tumor epithelial cell components showed ductule-like patterns composed of cuboidal to low-columnar cells (Fig. 2A-C). They were well to moderately differentiated, without microvascular or perineural invasion. Immunohistochemical staining showed diffuse positivity for $\mathrm{N}$-cadherin and focal positivity for neural cell adhesion molecule, but negative for S100 calcium-binding protein $\mathrm{P}$ in all cases. Alcian blue staining revealed that there was no intra- or extracellular mucin deposit in the tumor area. All cases were consistent with the small duct type (Fig. 2D-G) ${ }^{18}$ The non-tumor liver showed chronic liver disease, including four cases of $\mathrm{B}$ viral chronic hepatitis and a case of chronic alcoholic hepatitis (Table 3). In order to investigate the inflammatory response against the tumor, immune cell infiltration status was examined by immunohistochemical staining for $\mathrm{CD} 3$ (pan-T cell), CD4 (helper T cell), CD8 (cytotoxic T cell), FOXP3 (regulatory T cell), CD68 (macrophage), and CD163 (M2 macrophage) (Fig. $2 \mathrm{H}-\mathrm{K}$ and Supplementary Fig. 1). Extensive infiltration of $\mathrm{CD} 4^{+}, \mathrm{CD} 8^{+} \mathrm{T}$ cells and macrophages were found in both intratumoral and peritumoral areas. $\mathrm{FOXP}^{+} \mathrm{T}$ cells were present among $\mathrm{CD}^{+} \mathrm{T}$ cells with highest fraction of 10\% (Supplementary Table 1). Programmed death-ligand 1 was totally negative for both tumor and immune cells in all cases, indicating that infiltrated $\mathrm{T}$ cells are immunologically active on tumor cells. C-reactive protein was positive
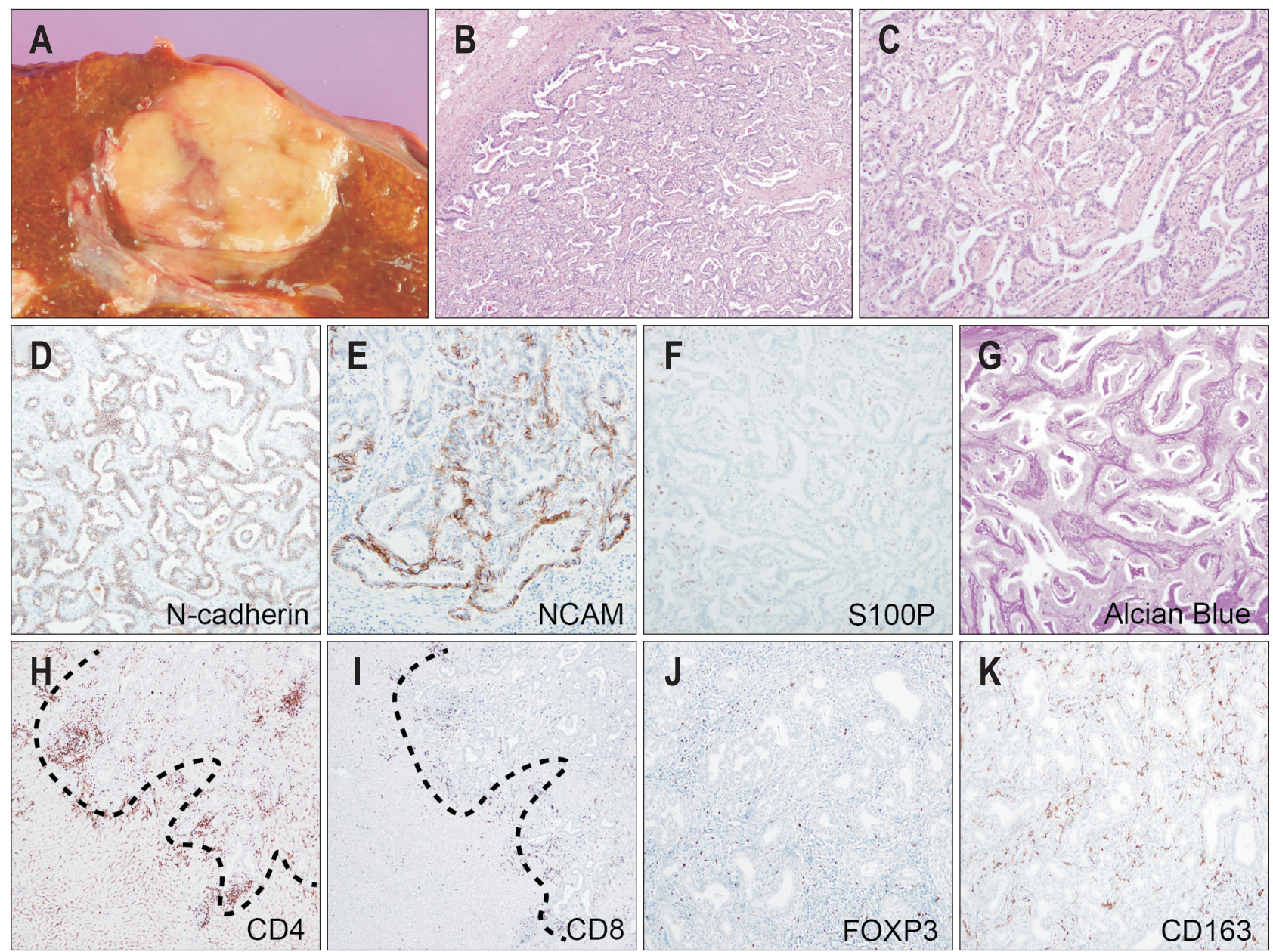

Fig. 2. Representative pathological features of intrahepatic cholangiocarcinoma (iCCA) with ductal plate malformation (DPM) pattern. (A) Representative gross image of an iCCA with predominant DPM pattern (case no. 1). The tumor mass had a yellow-tan solid appearance and was, relatively well-circumscribed without capsulation. (B,C) Microscopic images of iCCA with predominant DPM pattern (H\&E; $B, \times 40$ and $C, \times 100)$. (DF) The tumor epithelial component was positive for $\mathrm{N}$-cadherin (D), focally positive for neural cell adhesion molecule (E), and negative for S100 calcium-binding protein $P(F)$ by immunohistochemical staining $(\times 100)$. (G) Alcian blue staining showed absence of intra/extracellular mucin $(\times 100)$. $(\mathrm{H}-\mathrm{K})$ Representative immunohistochemical staining images of CD4 (H), CD8 (I), FOXP3 (J), and CD163 (K) (H and I, $\times 40$ and J and K, $\times 100)$. Rightupper side of the dotted lines of $(\mathrm{H})$ and $(\mathrm{I})$ indicate the tumor area. 
Table 3. Histopathological Features of the Five Cases with Intrahepatic Cholangiocarcinoma with Ductal Plate Malformation Pattern

\begin{tabular}{|c|c|c|c|c|c|c|c|c|}
\hline \multirow{2}{*}{$\begin{array}{l}\text { Case } \\
\text { No. }\end{array}$} & \multicolumn{5}{|c|}{ Gross and general microscopic characteristics } & \multicolumn{2}{|c|}{ Small \& large duct classification } & \multirow{2}{*}{$\begin{array}{c}\begin{array}{c}\text { Non-tumor } \\
\text { pathology }\end{array} \\
\text { Non-tumor liver }\end{array}$} \\
\hline & $\begin{array}{c}\text { Tumor size, } \\
\mathrm{cm}\end{array}$ & Gross type & $\begin{array}{c}\text { Tumor } \\
\text { differentiation }\end{array}$ & $\begin{array}{c}\text { Microvascular } \\
\text { invasion }\end{array}$ & $\begin{array}{l}\text { Perineural } \\
\text { invasion }\end{array}$ & $\begin{array}{l}\text { N-cadherin/NCAM/ } \\
\text { S100P/Alcian blue* }\end{array}$ & $\begin{array}{l}\text { Histological } \\
\text { type }\end{array}$ & \\
\hline 1 & 3.6 & Mass forming & Well differentiated & Absent & Absent & $++/+/-/-$ & Small duct & $\begin{array}{c}\text { Chronic B viral } \\
\text { hepatitis }\end{array}$ \\
\hline 2 & 2.0 & Mass forming & Well differentiated & Absent & Absent & $+/+/-/-$ & Small duct & $\begin{array}{l}\text { Chronic } \\
\text { alcoholic } \\
\text { hepatitis }\end{array}$ \\
\hline 3 & 1.5 & Mass forming & Moderately differentiated & Absent & Absent & $++/+/-/-$ & Small duct & $\begin{array}{c}\text { Chronic B viral } \\
\text { hepatitis }\end{array}$ \\
\hline 4 & 3.5 & Mass forming & Well differentiated & Absent & Absent & $++/+/-/-$ & Small duct & $\begin{array}{c}\text { Chronic B viral } \\
\text { hepatitis }\end{array}$ \\
\hline 5 & 3.0 & Mass forming & Moderately differentiated & Absent & Absent & $++/+/-/-$ & Small duct & $\begin{array}{c}\text { Chronic B viral } \\
\text { hepatitis }\end{array}$ \\
\hline
\end{tabular}

NCAM, neural cell adhesion molecule; S100P, S100 calcium-binding protein P.

${ }^{*++}$, diffuse positive stain; + , focal positive stain; - , negative stain.

in tumor cells of all cases, implying iCCAs with DPM pattern are inflammation class iCCAs. ${ }^{19,20}$ Non-tumor liver parenchyma showed periportal lymphocytic infiltration, a consistent finding with chronic hepatitis.

The follow-up period ranged from 22 to 122 months, during which all patients survived without evidence of tumor recurrence or metastasis. Noteworthy, cases no. 1 and no. 2 showed excellent outcomes, without recurrence or metastasis over 100 months. Case no. 1 was initially diagnosed as suspected HCC and received transarterial chemoinfusion 3 years prior to the surgery. After a 3-year follow-up, slight growth of the mass was noted, and the tumor was resected, which turned out to be an iCCA with a predominant DPM pattern. No evidence of previous chemotherapy effects, including tumor necrosis or fibrosis, was found during pathological examination.

\section{Massive parallel sequencing analysis of iCCAs with DPM pattern}

For DNA samples, the fraction of sequenced reads aligned to the reference genome was on average $97.4 \%$. The average mean target coverage was 547 across the samples, with the lowest being 273 for case no. 1 . The percentage of target regions that covered more than 100 times was above 92.2\% for all cases (mean: 94.3\%). For RNA samples, an average of $89.9 \%$ of sequenced reads, which is equivalent to 17.9 million reads per case, were on-target.

Analysis of the DNA samples included detection of missense or nonsense single nucleotide variants, in-frame or frameshift short insertions/deletions of the coding sequence, and mutations in splice donor/acceptor sites. In total, 64 genes were found to have somatic mutations among the five iCCA cases (Fig. 3), with an average of approximately 15 mutations per case. Missense mutations in FGFR2 and PTPRT were identified in three cases, and mutations in ARID1A, CDKN2A, IRS1, and KDM6A were identified in two cases. Two of the three FGFR2 variants identified were p.F276C, which was previously reported in iCCA. ${ }^{6}$ One of the two ARID1A variants was a frameshift variant (p.M1497fs), and one of the two CDKN2A variants was p.H83Y, which has been previously found in iCCA. Other mutations in 58 genes were sporadically found among the five patients. Mutations in well-known driver genes associated with iCCA, such as TP53, BAP1, ATM, $N F 1$, and STK11 were found to be present, whereas no mutations were found in other frequently mutated genes, such as IDH1, KRAS, and PBRM1 (Supplementary Table 2). In RNA analysis, case no. 2 was found to harbor the FGFR2-TNC fusion. No evidence of additional gene fusion was observed. Copy number alteration analysis of the DNA and RNA samples revealed no evidence of gene deletion or amplification in any of the five cases.

The clinico-radio-pathologic and genetic characteristics of iCCA with DPM pattern described above were compared with the descriptions for those of conventional iCCA in the previous literature, and were summarized in Table 4. ${ }^{13,18,21-25}$

\section{DISCUSSION}

iCCA with DPM pattern, in particular the one harboring the DPM pattern predominantly in tumor area, is a rare iCCA variant with unique histopathological features. The first and the largest report of iCCA with DPM pattern so far is a case series by Nakanuma et al. ${ }^{26}$ which included 10 cases, and this may reflect the low incidence of this subtype of iCCA. Herein, only five cases of iCCAs with DPM 


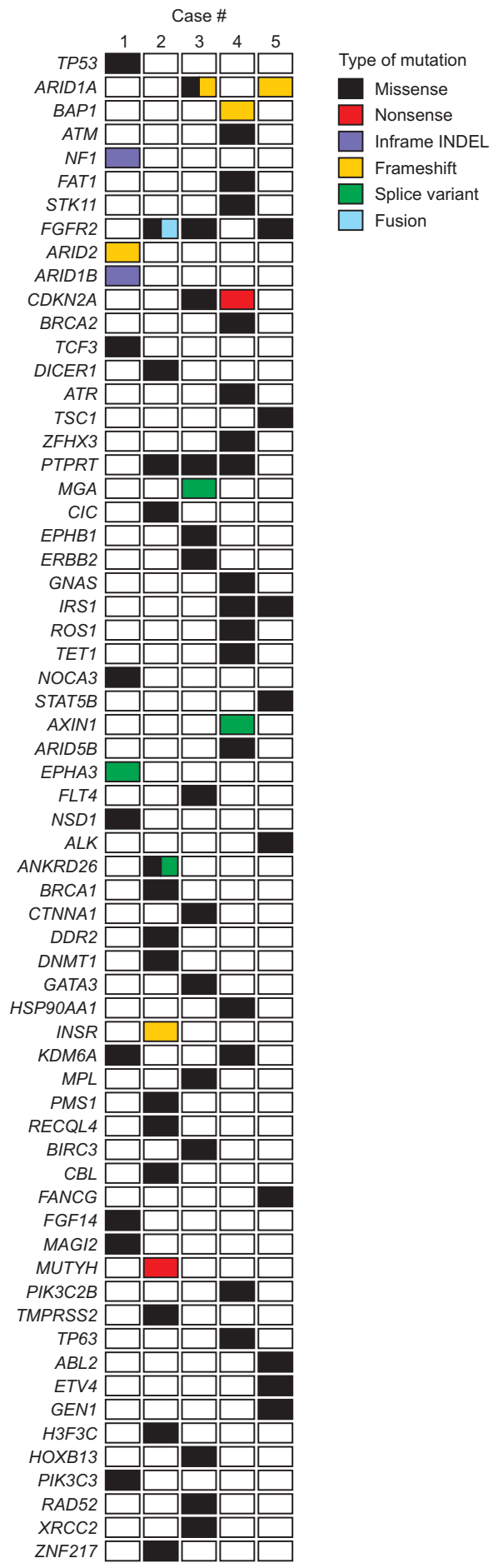

Fig. 3. Somatic mutation profiles of five patients with intrahepatic cholangiocarcinoma (iCCA) with ductal plate malformation pattern. Distribution of the identified somatic mutations in 64 genes across the five iCCA cases. The list of genes is ordered by the frequency of the mutations according to cBioPortal (https://www.cbioportal.org/) data regarding cholangiocarcinoma.

pattern were identified among 175 resected iCCAs, which makes its incidence rate lower than $3 \%$ among all iCCA cases. While the clinico-radio-pathologic and genetic char- acteristics of this rare tumor need investigation, its rarity is a limitation of our study. Recently, histopathological classification of iCCA of large duct and small duct types has been introduced. The large duct type, or bile duct type iCCA is usually composed of columnar tumor epithelial cells forming irregular-sized lumen with mucin production, whereas small duct type iCCA forms small tubular structures by low columnar or cuboidal tumor epithelial cells in a fibrous stroma, mostly with little or no mucin secretion. All five cases of iCCA with DPM pattern described herein were small duct type. ${ }^{18}$ These cases showed low aggressiveness in histopathology, with no microvascular or perineural invasion and absence of intrahepatic metastasis. No tumor recurrence or distant metastasis was observed in any of the cases during the follow-up period (22-122 months, mean: 67 months). Accordingly, small duct type iCCAs usually demonstrate longer postoperative survival than large duct type iCCAs. ${ }^{18,21}$

The predisposing factors for iCCA are known to be diverse, including chronic bile duct diseases such as parasitic infection, choledocholithiasis, primary sclerosing cholangitis, chronic hepatitis, and cirrhosis. ${ }^{27,28}$ Small duct type iCCAs are more commonly associated with chronic hepatitis and cirrhosis, compared with large duct type iCCAs. ${ }^{11,18,20}$ Interestingly, herein, the background liver of all patients showed chronic hepatitis related to hepatitis B virus and alcohol consumption. The $\mathrm{CD} 4^{+}$and $\mathrm{CD} 8^{+}$lymphocytic infiltration pattern shown in the present study did not reveal specific patterns. ${ }^{29} \mathrm{FOXP}^{+}$regulatory $\mathrm{T}$ cell fraction was lower than a previous study on HCC and iCCA, which states that high regulatory $\mathrm{T}$ cell fraction is associated with poor survival. ${ }^{30}$ The correlation between the presence of $\mathrm{CD}_{163}{ }^{+} \mathrm{M} 2$ macrophages and prognosis of iCCA is currently controversial and needs further study. ${ }^{31,32}$

In gadoxetic acid-enhanced MRI, iCCAs with DPM pattern commonly showed HCC-like imaging features, including non-rim arterial phase hyperenhancement (four cases, $80 \%$ ), enhancing capsule (one case, $20 \%$ ), and nonperipheral washout (two cases, $40 \%$ ). Imaging features favoring non-HCC hepatic malignancy were rarely observed in these patients, with only rim arterial phase hyperenhancement being observed in one case. As the iCCA with DPM pattern was concomitant with chronic hepatitis and common HCC-like imaging patterns, HCC was the primary radiologic impression in three cases $(60 \%)$, and LR-M (malignancy not specific for HCC) in two cases (40\%). Small duct type iCCA has been reported to frequently exhibit non-rim arterial phase hyperenhancement similar to HCC, and non-rim arterial phase hyperenhancement is known to be associated with good prognosis in iCCA. ${ }^{22,24,33}$ Another interesting imaging feature herein observed was 
Table 4. Comparison between Intrahepatic Cholangiocarcinoma (iCCA) with Ductal Plate Malformation Pattern and Conventional iCCA

\begin{tabular}{|c|c|c|c|}
\hline \multirow{2}{*}{ Features } & \multirow{2}{*}{$\begin{array}{l}\text { iCCA with DPM pattern } \\
\text { (small duct type) }\end{array}$} & \multicolumn{2}{|c|}{ Conventional iCCA } \\
\hline & & Small duct type & Large duct type \\
\hline \multicolumn{4}{|l|}{ Clinico-pathologic* } \\
\hline Male to female ratio & $0: 5$ & \multicolumn{2}{|c|}{ Slight male predominance } \\
\hline Differentiation & Well- to moderately differentiated & Well- to moderately differentiated & Moderately to poorly differentiated \\
\hline $\begin{array}{l}\text { Incidence of lymphovascular } \\
\text { invasion }\end{array}$ & $0 \%$ & \multicolumn{2}{|c|}{ Frequent } \\
\hline $\begin{array}{l}\text { Presence of chronic hepatitis } \\
\text { or cirrhosis }\end{array}$ & $100 \%$ & More frequent & Less frequent \\
\hline \multicolumn{4}{|l|}{ Radiologic $^{+}$} \\
\hline & Often non-rim AP hyperenhancement & Often non-rim AP hyperenhancement & $\begin{array}{r}\text { Rim hyperenhancement or } \\
\text { hypoenhancement in AP }\end{array}$ \\
\hline & Absence of bile duct involvement & Absence of bile duct involvement & $\begin{array}{l}\text { Bile duct dilatation at tumor } \\
\text { periphery } \\
\text { Bile duct encasement of tumor } \\
\text { Periductal tumor infiltration }\end{array}$ \\
\hline & Lobulated/round contour & Lobulated/round contour & Irregular contour \\
\hline & Absence of lymph node enlargement & Absence of lymph node enlargement & Lymph node enlargement \\
\hline \multicolumn{4}{|c|}{ 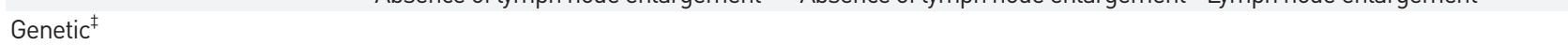 } \\
\hline $\begin{array}{l}\text { Frequently mutated genes } \\
\text { (frequency of occurrence } \\
\text { in the present study, \%) }\end{array}$ & $\begin{array}{l}\text { FGFR2 (60) } \\
\text { PTPRT (60) } \\
\text { ARID1A (40) } \\
\text { CDKN2A (40) } \\
\text { IRS1 (40) } \\
\text { KDM6A (40) } \\
\text { TP53 (20) } \\
\text { BAP1 (20) } \\
\text { ATM (20) } \\
\text { NF1 (20) } \\
\text { STK11 (20) } \\
\text { FGFR2 fusion (20) }\end{array}$ & $\begin{array}{l}\text { Frequent IDH1/2 mutation } \\
\text { Frequent } F G F R 2 \text { fusion }^{\S}\end{array}$ & $\begin{array}{l}\text { Frequent KRAS mutation } \\
\text { Frequent TP53 mutation }\end{array}$ \\
\hline
\end{tabular}

DPM, ductal plate malformation; AP, arterial phase.

*Mass-forming type iCCA is regarded as conventional iCCA. Clinico-pathological characteristics of conventional iCCA were summarized from previous literature ${ }^{18,21} ;{ }^{\dagger}$ Radiologic findings of conventional iCCA were summarized from previous literature ${ }^{22-25} ;{ }^{\ddagger}$ Genetic features of conventional iCCA were summarized from previous literature ${ }^{13} ;{ }^{\S}$ Mutations that were also found in the present study.

frequent peritumoral shunt, which was detected in the arterial phase and/or portal phase in four cases (80\%). Previously, peritumoral enhancement was reported in cholangiolocarcinoma, a histological variant belonging to small duct type iCCA, and communication between tumoral sinusoids and portal venules was suggested as the cause. ${ }^{34}$

The mutational profile of iCCA has been actively investigated in recent years, which revealed its highly heterogeneous nature with respect to its genomic, transcriptomic, and epigenomic landscape. ${ }^{7}$ iCCA has substantial mutational heterogeneity; nevertheless, iCCA exhibits less frequent KRAS and SMAD4 mutations than extrahepatic $\mathrm{CCA}$, and $I D H 1 / 2$ mutations are almost exclusively found in iCCA. ${ }^{35,36}$ According to the histological subtypes of iCCA, large duct type iCCA tend to follow the mutational profile of extrahepatic CCA, whereas small duct type iCCA shows frequent $I D H 1 / 2$ mutations (about $20 \%$ in a recent report $^{37}$ ) and FGFR2 fusions (10\% to 30\% in multiple reports). ${ }^{10,12,18,38}$ Recent advances in high-throughput nucleic acid sequencing and worldwide large cohort analyses revealed several frequently mutated genes associated with iCCA, namely TP53, IDH1/2, tyrosine kinase pathwayrelated genes including KRAS, SMAD4 and FGFR, and chromatin-remodeling-related genes including ARID1A, $P B R M 1$ and $B A P 1{ }^{7,9,39,40}$ In the present study, three patients showed single nucleotide variants in FGFR2, among whom one patient harbored a fusion of FGFR2 with TNC. The clinicopathological significance of single nucleotide variants in the coding regions of FGFR2 warrants further investigation, and TNC as a fusion counterpart for FGFR2 in iCCA has not been reported to date. Another most frequently mutated gene in this study (three out of five cases) was PTPRT. Mutations in this gene have been reported to be related to STAT3 activation in head and neck cancers. ${ }^{41}$ ARIDIA was mutated in two patients, which is consistent with a previous report by Sasaki et al. ${ }^{42}$ showing that genetic alterations of ARIDIA are associated with iCCA with DPM pattern. These results loosely follow those of 
Clusters 1 and 4 proposed by Jusakul et al., ${ }^{39}$ which were characterized by ARID1A and FGFR family mutations, respectively. On the other hand, iCCA can be classified as inflammation and proliferation classes; inflammation class overlaps with small duct type and shows good outcome, while proliferation class is associated with large duct type and shows worse outcome than inflammation class. ${ }^{19-21}$ The cases presented in this study can be classified into inflammation class, as those are all small duct type iCCAs, C-reactive protein-positive and characteristic genetic variants usually found in proliferation class including KRAS was absent. This may partly explain the excellent outcome of iCCA with DPM pattern. However, the correlation between the absence of $I D H 1 / 2$ mutation, which are frequently found in small duct type iCCAs, and iCCA with DPM pattern needs further investigation. ${ }^{11,12,37}$ Overall, the mutational profiles of iCCA with predominant DPM pattern are somewhat heterogeneous, and subsequent studies are required to reveal epigenomic characteristics of iCCA with DPM pattern, including DNA methylation analysis.

In summary, this study reports the clinicopathological, radiological, and molecular characteristics of iCCA with DPM pattern. To the best of our knowledge, this is the first study to investigate the genetic landscape of iCCA with DPM pattern using massive parallel sequencing. The integrated patho-radio-molecular findings suggest that iCCA with DPM pattern is a specific subtype of small duct type iCCA.

\section{CONFLICTS OF INTEREST}

No potential conflict of interest relevant to this article was reported.

\section{ACKNOWLEDGEMENTS}

This research was supported by the basic science research program of the National Research Foundation of Korea (NRF) (NRF-2020R1A2B5B01001646), and by the Bio and Medical Technology Development Program of the NRF funded by the Ministry of Science and ICT (NRF2016M3A9D5A01952416).

We thank Mr. Won Young Park (Severance Hospital, Seoul, Korea) for the technical support during the sample preparation and next-generation sequencing operations.

\section{AUTHOR CONTRIBUTIONS}

Study conception and design: T.C., H.R., J.E.Y., G.H.C., H.K., H.S.S., Y.N.P. Data acquisition: T.C., H.R., J.E.Y., H.S.S., Y.N.P. Data analysis and interpretation: T.C., H.R. Drafting of the manuscript: T.C., H.R., Y.N.P. Technical and material support: J.E.Y., G.H.C., H.K., H.S.S. Funding acquisition and study supervision: Y.N.P. Approval of final manuscript: all authors.

\section{ORCID}

Taek Chung Hyungjin Rhee https://orcid.org/0000-0001-7567-0680 Hyo Sup Shim https://orcid.org/0000-0001-7759-4458 Jeong Eun Yoo Gi Hong Choi https://orcid.org/0000-0002-5718-3624 https://orcid.org/0000-0001-9990-279X https://orcid.org/0000-0002-1593-3773 Haeryoung Kim https://orcid.org/0000-0002-4205-9081 Young Nyun Park https://orcid.org/0000-0003-0357-7967

\section{SUPPLEMENTARY MATERIALS}

Supplementary materials can be accessed at https://doi. org/10.5009/gnl210174.

\section{REFERENCES}

1. Massarweh NN, El-Serag HB. Epidemiology of hepatocellular carcinoma and intrahepatic cholangiocarcinoma. Cancer Control 2017;24:1073274817729245.

2. Nakanuma Y, Sato Y, Ikeda H, et al. Intrahepatic cholangiocarcinoma with predominant "ductal plate malformation" pattern: a new subtype. Am J Surg Pathol 2012;36:16291635.

3. Desmet VJ. Congenital diseases of intrahepatic bile ducts: variations on the theme "ductal plate malformation". Hepatology 1992;16:1069-1083.

4. Awasthi A, Das A, Srinivasan R, Joshi K. Morphological and immunohistochemical analysis of ductal plate malformation: correlation with fetal liver. Histopathology 2004;45:260-267.

5. WHO Classification of Tumours Editorial Board. Digestive system tumours. 5th ed. Lyon: International Agency for Research on Cancer, 2019.

6. Jiao Y, Pawlik TM, Anders RA, et al. Exome sequencing identifies frequent inactivating mutations in BAP1, ARID1A and PBRM1 in intrahepatic cholangiocarcinomas. Nat Genet 2013;45:1470-1473.

7. Farshidfar F, Zheng S, Gingras MC, et al. Integrative genom- 
ic analysis of cholangiocarcinoma identifies distinct IDHmutant molecular profiles. Cell Rep 2017;18:2780-2794.

8. Zou S, Li J, Zhou H, et al. Mutational landscape of intrahepatic cholangiocarcinoma. Nat Commun 2014;5:5696.

9. Lowery MA, Ptashkin R, Jordan E, et al. Comprehensive molecular profiling of intrahepatic and extrahepatic cholangiocarcinomas: potential targets for intervention. Clin Cancer Res 2018;24:4154-4161.

10. Goeppert B, Toth R, Singer S, et al. Integrative analysis defines distinct prognostic subgroups of intrahepatic cholangiocarcinoma. Hepatology 2019;69:2091-2106.

11. Liau JY, Tsai JH, Yuan RH, Chang CN, Lee HJ, Jeng YM. Morphological subclassification of intrahepatic cholangiocarcinoma: etiological, clinicopathological, and molecular features. Mod Pathol 2014;27:1163-1173.

12. Wang T, Drill E, Vakiani E, et al. Distinct histomorphological features are associated with IDH1 mutation in intrahepatic cholangiocarcinoma. Hum Pathol 2019;91:19-25.

13. Banales JM, Marin JJG, Lamarca A, et al. Cholangiocarcinoma 2020: the next horizon in mechanisms and management. Nat Rev Gastroenterol Hepatol 2020;17:557-588.

14. American College of Radiology. Liver Imaging Reporting and Data System (LI-RADS) version 2018 [Internet]. Reston: American College of Radiology; c2018 [cited 2020 Oct 10]. Available from: https://www.acr.org/Clinical-Resources/ Reporting-and-Data-Systems/LI-RADS/CT-MRI-LI-RADSv2018.

15. Bankhead P, Loughrey MB, Fernández JA, et al. QuPath: open source software for digital pathology image analysis. Sci Rep 2017;7:16878.

16. Li H, Durbin R. Fast and accurate short read alignment with Burrows-Wheeler transform. Bioinformatics 2009;25:17541760.

17. Dunn T, Berry G, Emig-Agius D, et al. Pisces: an accurate and versatile variant caller for somatic and germline nextgeneration sequencing data. Bioinformatics 2019;35:15791581.

18. Hayashi A, Misumi K, Shibahara J, et al. Distinct clinicopathologic and genetic features of 2 histologic subtypes of intrahepatic cholangiocarcinoma. Am J Surg Pathol 2016;40:1021-1030.

19. Sia D, Hoshida Y, Villanueva A, et al. Integrative molecular analysis of intrahepatic cholangiocarcinoma reveals 2 classes that have different outcomes. Gastroenterology 2013;144:829-840.

20. Rhee H, Ko JE, Chung T, et al. Transcriptomic and histopathological analysis of cholangiolocellular differentiation trait in intrahepatic cholangiocarcinoma. Liver Int 2018;38:113-124.

21. Chung T, Rhee H, Nahm JH, et al. Clinicopathological characteristics of intrahepatic cholangiocarcinoma according to gross morphologic type: cholangiolocellular differentiation traits and inflammation- and proliferation-phenotypes. HPB (Oxford) 2020;22:864-873.

22. Rhee H, Kim MJ, Park YN, An C. A proposal of imaging classification of intrahepatic mass-forming cholangiocarcinoma into ductal and parenchymal types: clinicopathologic significance. Eur Radiol 2019;29:3111-3121.

23. Nam JG, Lee JM, Joo I, et al. Intrahepatic mass-forming cholangiocarcinoma: relationship between computed tomography characteristics and histological subtypes. J Comput Assist Tomogr 2018;42:340-349.

24. Fujita N, Asayama Y, Nishie A, et al. Mass-forming intrahepatic cholangiocarcinoma: enhancement patterns in the arterial phase of dynamic hepatic CT: correlation with clinicopathological findings. Eur Radiol 2017;27:498-506.

25. Kim SA, Lee JM, Lee KB, et al. Intrahepatic mass-forming cholangiocarcinomas: enhancement patterns at multiphasic CT, with special emphasis on arterial enhancement pattern: correlation with clinicopathologic findings. Radiology 2011;260:148-157.

26. Nakanuma Y, Sato Y, Harada K, Sasaki M, Xu J, Ikeda H. Pathological classification of intrahepatic cholangiocarcinoma based on a new concept. World J Hepatol 2010;2:419427.

27. Banales JM, Cardinale V, Carpino G, et al. Expert consensus document: cholangiocarcinoma: current knowledge and future perspectives consensus statement from the European Network for the Study of Cholangiocarcinoma (ENS-CCA). Nat Rev Gastroenterol Hepatol 2016;13:261-280.

28. Palmer WC, Patel T. Are common factors involved in the pathogenesis of primary liver cancers? A meta-analysis of risk factors for intrahepatic cholangiocarcinoma. J Hepatol 2012;57:69-76.

29. Kasper HU, Drebber U, Stippel DL, Dienes HP, Gillessen A. Liver tumor infiltrating lymphocytes: comparison of hepatocellular and cholangiolar carcinoma. World J Gastroenterol 2009;15:5053-5057.

30. Kobayashi N, Hiraoka N, Yamagami W, et al. FOXP3+ regulatory $\mathrm{T}$ cells affect the development and progression of hepatocarcinogenesis. Clin Cancer Res 2007;13:902-911.

31. Hasita H, Komohara Y, Okabe H, et al. Significance of alternatively activated macrophages in patients with intrahepatic cholangiocarcinoma. Cancer Sci 2010;101:1913-1919.

32. Miura T, Yoshizawa T, Hirai H, et al. Prognostic impact of CD163+ macrophages in tumor stroma and CD8+ T-Cells in cancer cell nests in invasive extrahepatic bile duct cancer. Anticancer Res 2017;37:183-190.

33. Min JH, Kim YK, Choi SY, et al. Intrahepatic mass-forming cholangiocarcinoma: arterial enhancement patterns at MRI and prognosis. Radiology 2019;290:691-699.

34. Kozaka K, Matsui O, Kobayashi S, et al. Dynamic CT find- 
ings of cholangiolocellular carcinoma: correlation with angiography-assisted CT and histopathology. Abdom Radiol (NY) 2017;42:861-869.

35. Churi CR, Shroff R, Wang Y, et al. Mutation profiling in cholangiocarcinoma: prognostic and therapeutic implications. PLoS One 2014;9:e115383.

36. Tsai JH, Huang WC, Kuo KT, Yuan RH, Chen YL, Jeng YM. S100P immunostaining identifies a subset of peripheral-type intrahepatic cholangiocarcinomas with morphological and molecular features similar to those of perihilar and extrahepatic cholangiocarcinomas. Histopathology 2012;61:11061116.

37. Ma B, Meng H, Tian Y, et al. Distinct clinical and prognostic implication of IDH1/2 mutation and other most frequent mutations in large duct and small duct subtypes of intrahepatic cholangiocarcinoma. BMC Cancer 2020;20:318.

38. Kendall T, Verheij J, Gaudio E, et al. Anatomical, histomor- phological and molecular classification of cholangiocarcinoma. Liver Int 2019;39 Suppl 1:7-18.

39. Jusakul A, Cutcutache I, Yong $\mathrm{CH}$, et al. Whole-genome and epigenomic landscapes of etiologically distinct subtypes of cholangiocarcinoma. Cancer Discov 2017;7:1116-1135.

40. Cardinale V, Bragazzi MC, Carpino G, et al. Intrahepatic cholangiocarcinoma: review and update. Hepatoma Res 2018;4:20.

41. Lui VW, Peyser ND, Ng PK, et al. Frequent mutation of receptor protein tyrosine phosphatases provides a mechanism for STAT3 hyperactivation in head and neck cancer. Proc Natl Acad Sci U S A 2014;111:1114-1119.

42. Sasaki M, Sato Y, Nakanuma Y. Cholangiolocellular carcinoma with "ductal plate malformation" pattern may be characterized by ARID1A genetic alterations. Am J Surg Pathol 2019;43:352-360. 